\title{
News for 2014
}

\section{Domenico Prisco}

(C) SIMI 2014

Dear Readers,

some news for IEM in 2014!

The first one item is that I have been appointed as Editor-in-chief for 4 more years by the Board of the Italian Society of Internal Medicine. I thank the President Gino Roberto Corazza and all the board for their appreciation of the efforts done for IEM over the past few years.

In 2013, we have had the opportunity to publish more papers, especially original contributions, due to the increase from 6 to 8 issues published per year. The time the accepted papers stay in an "online first" state has decreased, and in particular we have published all the papers accepted in 2011 and many of those accepted in 2012. Our goal is to have no paper in the "online first" state for more than 10-12 months.

One year ago I wrote that a principal objective for 2013 was that the printed journal would have been sent to our subscribers only upon request and with a small charge. The requests for the printed journal were just a few which confirms that most readers prefer the online journal rather than the printed version.

Welcome to the new members of our Editorial Boards and a warm thank you to those who completed their term in 2013.
In 2014, according to the new instructions, we will stop accepting case reports. We will continue to publish case records upon invitation (with Nicola Montano as supervisor), and to consider Medical Illustrations freely submitted (with Peter Rosen as supervisor). Letters to the editor should be first of all comments on material published in IEM.

The section of Emergency Medicine, led by Peter Rosen, is very active and received many submissions. The quality of these submissions has importantly improved over the past few years, and you will soon read more papers in this field.

The Clinical Evidence section has been modified. The deputy editor Lorenzo Moja, due to his relevant engagements, has left his place to Giorgio Costantino and Giovanni Casazza who will help the editor Gian Franco Gensini to improve the section with new ideas and new coworkers. Thank you, Lorenzo and welcome, Giorgio and Giovanni. Finally, the cutting edge papers, under the supervision of Nicola Montano, will be more regularly published.

We hope you continue to enjoy and support our journal, and wish you a wonderful 2014 of health and active work.

D. Prisco $(\bowtie)$

Florence, Italy

e-mail: priscod@aou-careggi.toscana.it 